\section{Applying electrodynamics to astrophysics}

\section{Rowan-Robinson}

Theoretical Physics and Astrophysics. By V.L. Ginzburg. Pp.464. (Pergamon: Oxford, 1979.) Hardback £25; paperback $£ 10$.

PROFESSOR ter Haar has performed the arduous task of translating this advanced textbook by a prominent Russian astrophysicist admirably. Impressive though this book is in several respects, however, I feel it needed a good deal more work on it before it was published in a Student Edition.

A more accurate title for the book would have been $A$ Treatise on Electrodynamics, with some Applications to Astrophysics.
Most of the book consists of rather academic discourses on the themes of those great books by Landau and Lifschitz. However, Ginzburg does not have quite the same ability to illuminate abstract principles with simple and concrete examples. The absence of any sub-headings within the chapters makes the book hard to read and limits its usefulness as a work of reference. The neglect of the Western literature on the subject in the references cited (over $80 \%$ of the references are Russian and about a third are to papers by Ginzburg and coworkers) will not commend the book to other workers in the field, despite Ginzburg's plea in the Preface to the English edition that this was not so noticeable in the Russian edition and that the book was based on a lecture course. It is precisely this kind of deficiency in a lecture course that one expects to be made good in a textbook. The publishers have managed entirely to omit the first four pages of Chapter 3.

The most interesting and useful chapters for the general astrophysicist will probably be Chapter 5 on synchrotron radiation and Chapters 15-17 on cosmic-ray, X-ray and gamma-ray astronomy, though the review of the observational situation in these latter areas is rather out of date (the Russian edition appeared in 1974). Not all the formulae given are of sufficient accuracy for contemporary astrophysical applications. In the formulae for bremsstrahlung, for example, the Gaunt factor is assumed to be unity: some space could surely have been found for a discussion of the exact value.

The book remains, however, an important one by a major physicist. The specialist in electrodynamics will find it a useful review of Russian work in the field and the theorist in high-energy astrophysics will find some parts of the book valuable.

Michael Rowan-Robinson is Reader in Astronomy at Queen Mary College, London, UK, and Gresham Professor of Astronomy at the City University.

\section{Introducing meteorology}

\section{J.S.A. Green}

Fundamentals of Meteorology. By L.J. Battan. Pp.321. (Prentice-Hall: Hemel Hempstead, UK, 1979.) £10.90. The Atmosphere. By Lutgen and Tarbuck. Pp.413. (Prentice-Hall: Hemel Hempstead, UK, 1979.) £10.90.

Most of us have a vested interest in meteorology, and any books like those reviewed here, that seek to explain the intricate details of the subject to a wider audience, are always welcome. These two books summarise modern meteorology for the 'non-mathematician', by which the authors mean one not familiar with 'differential calculus'. Both books cover many topics of interest to a general reader, such as air pollution (supersonic airliners come in for substantial unquantitative criticism), clouds, the generation of rain, storms and climate. Curiously, their contents overlap by some $75 \%$. To a scientist used to a uniform system of units, they both show a wide variety. One has a useful table of densities of common substances expressed in $\mathrm{g} \mathrm{cm}^{-3}$, as well as in $\mathrm{kg} \mathrm{m}^{-3}$. That the figures in one column are just 1,000 times the figures in the other might well strike the unitiated as an odd thing for scientists to pay attention to. A variety of basic units is used: calories, watts, feet, metres, knots, metres per second. Presumably the aim is to use units familiar to the reader, but one loses something of scientific nicety. The literary style is inexorable rather than objectionable: " . . . precipitation data for localities in selected precipitation regimes ..."' is typical.

My only major reservation concerns the sort of information that the authors have tried to present. A typical, but not unimportant, topic concerns the geostrophic wind. This blows along the lines of equal pressure, not across them from high to low pressure, as one might naively suppose. If I had to start from my first sight of Newton's laws of motion in order to arrive at this result, then I think I would feel cheated. The important fact is that the Coriolis acceleration is at rightangles to, and proportional to, the wind and not as one would expect, along the direction of the change in velocity as we usually measure it relative to the Earth. The point is made by having an experimenter, who used to be a golfer (but who now uses intercontinental ballistic missiles), fire missiles from the North Pole while the rest of the Earth moves past relative to the missile. But this rightly leaves me unimpressed because the golfer is in a situation where the real acceleration of the missile is zero, and the apparent acceleration seen from the Earth is fictitious. For geostrophic motion where the Coriolis acceleration balances the force due to the pressure field, it is exactly the other way round: the relative acceleration is small but the real (Coriolis) acceleration is large. Thus, trying to represent what really happens in terms that are too simple, may mislead that thoughtful nonmathematician. It might be better to tell him that geostrophic balance happens and that it is because of the rotation of the
Earth. However, if you go and study mathematics, you can find the inevitable connection between the Newtonian laws and the geostrophic wind.

One expects to find authors representing such differences in philosophy that this topic highlights, and the books do summarise much modern meteorology, with particular emphasis on conditions in North America. Certainly one (but not both) would be a useful teaching aid for any non-meteorologist wishing to give a course on meteorology to American undergraduates. They are a useful Introduction to Meteorology, with the mathematical bits left out, and nicely illustrated by satellite pictures. To me they seemed to lack something of the enthusiasm of the authors for their subject. One of them recommends Minnaert's book, Light and Colour in the Open Air, to those interested in optics. Minnaert starts his book: "A lover of Nature responds to her phenomena as naturally as he breathes and lives." Now that is the sort of attitude I think we can expect to put before this person who has failed to acquire 'differential calculus' amongst his grades, and it does not come over to me from either of these texts.

Let us hope that their readers will absorb the philosophy and enthusiasm of Minnaert, as well as the useful factual information which he will find in these books.

J.S.A. Green is Reader in Physical Climatology at Imperial College, University of London, UK and Head of a Group teaching meteorology at MSc. level to students with a first degree in mathematics or physics. 